SCIREA Journal of Medicine

http://www.scirea.org/journal/PM

April 4, 2021

Volume 5, Issue 1, February 2021

\title{
PRINCIPLES OF THE STRUCTURAL ORGANIZATION OF THE HUMAN HEART
}

\section{Spirina G.A.}

Ural State Medical University, Department of Human Anatomy, Yekaterinburg, Russian Federation

\begin{abstract}
One of the important areas of research in biological development is the study of the laws and algorithms of organ shaping. The phenomenon of symmetry in the forms of biological bodies is considered as the main provision on the existence of specific laws of morphogenesis. A morphometric study of the parameters of the heart, parts of the ventricles, and parts of the interventricular septum was carried out on 545 human heart preparations (from fetuses of 1232 weeks to the age of 90 years). It has been established that two types of proportions are realized in the structure of the heart: affine (simple) relations of two anatomical dimensions of the heart chambers and Wurf proportions. The laws of conformal symmetry are manifested in all age periods and not only in the relationship between the external parameters of the heart, but also in the relationship of anatomical structures inside the heart chambers. The presented data on the subordination of the anatomical structures of the heart to the laws of conformal symmetry should be considered in close connection with their individual and age-related variability.
\end{abstract}


Keywords: heart, structural organization, conformal symmetry.

\section{Introduction}

The question of general biological properties of morphogenesis is one of the most urgent, as it testifies to the unity of living nature. The question of general biological properties of morphogenesis is one of the most actual, as it testifies to the unity of living nature. The study of biosymmetry is useful for solving the issues of harmonization in the systems man machine - environment (Petukhov S.V., 1988). The deepening of biological knowledge is accompanied by the discovery of more and more facts of subordination to the principles of conformal symmetry of various biological objects that differ in scale and level of organization. The study of the laws and algorithms of organic morphogenesis is one of the important directions in the study of biological development. There are two approaches to studying the shape of an internal organ.. In one of them, the form is studied only from the point of view of its correspondence to the function and conditions of the external environment. Another approach is based on the recognition that the structuring of biological bodies is not completely determined by environmental conditions and function. The phenomenon of symmetry in the forms of biological bodies is considered as the basic position on the existence of specific laws of morphogenesis. According to S.V. Petukhov $(1981,1988)$ in the variety of forms there is an ordering independent of the function, which obeys the mathematical laws of harmony. According to I.I. Schmalhausen (1935), most of the morphogenetic reactions are clearly "expedient" in nature - they seem to provide consistency in the location and relative size of organs. The works of S.V. Petukhova (1981, 1988), R.T. Nigmatullina et al. (2003) found that three-member kinematic blocks prevail in the structure of the human body. The criterion for conformal symmetry between blocks of three consecutive line segments is "wurf". All the three-member kinematic chains of the human body obey the rule of the "golden wurf" (Petukhov S.V., 2004). From the moment of birth of an individual, the growth of the body occurs with the preservation of the size of the wurf of each three-term kinematic block. "Golden Wurf" (1.309) is the "canon" of the human body, its proportions are especially aesthetically pleasing, widely implemented in the human body.

Research Objective - study of the structural organization of the human heart in ontogenesis. 
The material of the work included 545 heart preparations from fetuses 12 - 32 weeks up to the age of 90 years, who died from accidents or somatic diseases in the absence of heart defects, lung diseases, kidneys. A morphometric study of the parameters of the heart, parts of the ventricles, parts of the interventricular septum was performed. Knowledge of the quantitative ratios of intracardiac structures in a normally formed heart is necessary for the implementation of surgical interventions aimed at their restoration. At the same time, the indicators of the inflow and outflow sections of the ventricles are used to assess hypertrophy and dilatation of the heart cavities. When analyzing data on the quantitative anatomy of the heart and its chambers in people of different ages, we proceeded from the assumptions of S.V. Petukhova (1981) that the mysteries of the formation of biological bodies lie in the conformal nature of space. By analogy with the kinematic diagram of the structure of the musculoskeletal system, the parameters of the heart of people of different ages, the length of the inflow and outflow sections of the ventricles, their total width can be represented as a three-membered block. As the proportions of this block grow, they change according to the rules of conformal transformations, that is, the change in one parameter is consistent with the change in the other two so that in the straightened block the invariant of one-dimensional circular formations - the wurf - remains unchanged.

$$
\mathrm{W}=\frac{(C-A) \cdot(D-B)}{(C-B) \cdot(D-A)}
$$
A. $\mathrm{B}$ $\mathrm{C}$ $\mathrm{D}$

W - wurf

A - B - heart length

B - C - heart width

C - D - heart thickness

In fetuses of different ages, three heart shapes were identified: a wide short, long narrow, and a transitional heart. Regardless of the shape of the heart and the period of development of the wurfs, the parameters of the heart are close in value (Table 1). 
Table 1. Wurf value (W) of human fetal heart, rel. units

\begin{tabular}{|c|c|}
\hline Fetal age (weeks) & Wurf value, rel. \\
\hline 16 & 1,25 \\
\hline 20 & 1,27 \\
\hline 24 & 1,21 \\
\hline 28 & 1,23 \\
\hline 32 & 1,22 \\
\hline
\end{tabular}

For comparison, the cardiac parameters were calculated for the wurfs in the postnatal period of ontogenesis (Table 2).

Table 2. Values of wurfs (W) of the human heart in the postnatal period of ontogenesis, rel. units

\begin{tabular}{|l|l|}
\hline Age periods & Wurf value, rel. \\
\hline Newborns & 1,27 \\
\hline Infant age & 1,29 \\
\hline Early childhood & 1,23 \\
\hline First childhood & 1,22 \\
\hline Second childhood & 1,21 \\
\hline Adolescent age & 1,23 \\
\hline Youthful age & 1,23 \\
\hline Mature age & 1,23 \\
\hline I period & 1,21 \\
\hline II period & 1,23 \\
\hline Elderly age & 1,22 \\
\hline Old age & \\
\hline
\end{tabular}

The solution of health problems is unthinkable without a deep study of the basic laws of individual development and clarification of the mechanisms that regulate the functions of organs in the pre- and postnatal periods of ontogenesis. The study of the morphogenesis of the heart and the formation of the shape of the organ at the early stages of development is not only a theoretical, but also a great practical problem. Knowledge of the dynamics of organ development and structural features at different times of the prenatal period allows the doctor 
to correct the developmental pathology. To describe the possible growth transformation of the ventricles of the fetal heart, a formula was used to calculate the wurfs, where A - B is the length of the ventricular inflow section, B - C is the length of the ventricular outflow section, $\mathrm{C}-\mathrm{D}$ is the total width of the ventricular inflow and outflow sections. Wurf denotes a value expressing the ratio of three conformally symmetric blocks (segments or members of a numerical sequence) connected by one of the types of circular symmetry. The ordered arrangement of the values of the length of the inflow and outflow sections of the ventricles, their width made up such a numerical sequence in which $1,4,7,10 \ldots$ its members were equal to the length of the inflow section; $2,5,8,11 \ldots$ members were equal to the length of the outflow section; $3,6,9,12 \ldots$ members - the total width of these sections. Three adjacent numbers of the sequence were considered as the lengths of three segments, which were used in the geometric formula to calculate the value of the wurf. The average value of the ventricular wurf, calculated for fetuses of 16 - 32 weeks, ranges from 1.24 to 1.3, approaching the "golden" wurf (1.309). The ratio of the length of the inflow and outflow sections, their total width for each ventricle is relatively constant, does not depend on the gestational age. Wurfs of the right and left ventricles are close in value (Table 3). Similar values of the heart ventricle wurfs were revealed by us according to the results of the study in the postnatal period of ontogenesis (Table 4).

Table 3. Average value of wurfs (W) of the ventricles of the heart of human fetuses 16 - 32 weeks, rel. units

\begin{tabular}{|c|c|c|c|c|}
\hline Age & \multicolumn{2}{|c|}{ Right ventricle } & \multicolumn{2}{|c|}{ Left ventricle } \\
\hline Fetal age (weeks) & $\begin{array}{l}\text { Wurf } \\
\text { from }\end{array}$ & rf deviation & $\begin{array}{l}\text { Wur } \\
\text { from }\end{array}$ & Wurf deviation \\
\hline 16 & 1,25 & 4,58 & 1,26 & 3,82 \\
\hline 20 & 1,25 & 4,58 & 1,26 & 3,82 \\
\hline 24 & 1,25 & 4,58 & 1,24 & 5,3 \\
\hline 28 & 1,25 & 4,58 & 1,24 & 5,3 \\
\hline 32 & 1,27 & 3,05 & 1,24 & 5,3 \\
\hline
\end{tabular}


Table 4. Average value of wurfs (W) of human heart ventricles in the postnatal period of ontogenesis, rel. units

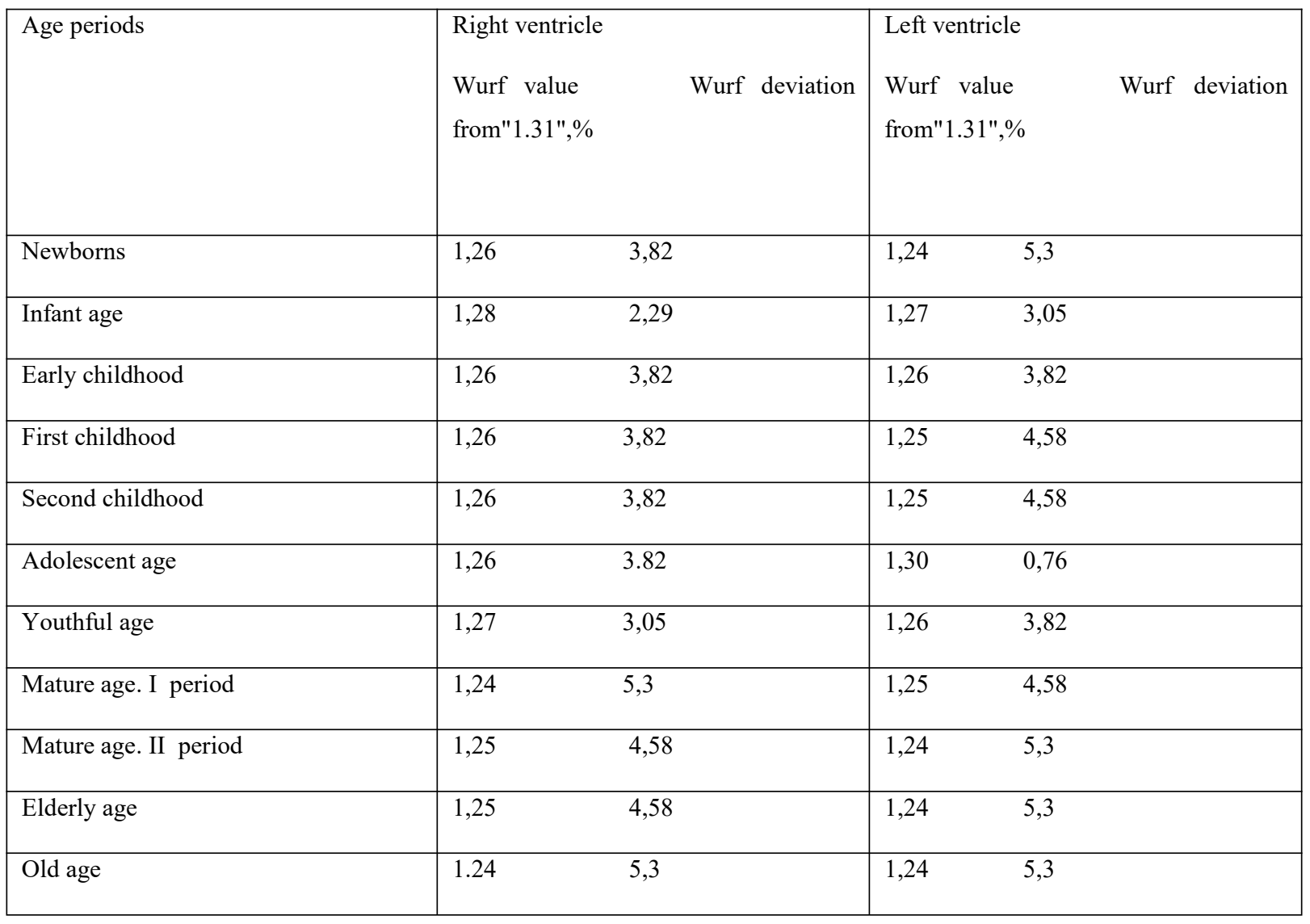

In fetuses from 12 weeks of intrauterine development, the ratio between the length of the ventricular inflow and outflow sections is similar to those in the postnatal period of ontogenesis. The dynamics of changes in the length of the inflow and outflow sections of the fetal heart ventricles corresponds to the parabolic type of growth. The proportions of the ratio of the dimensions of the length, width of the inflow and outflow sections of the ventricles, as well as the parameters of the heart as a whole, as they grow, change according to the rules of conformal symmetry, namely, in each age period, the lengthening of one of the dimensions is coordinated with the change in the other two dimensions so that the ratio as the length, width of the inflow and outflow sections of the ventricles, so the length, width, thickness of the whole heart is close to 1.31 . The manifestation of the principles of conformal symmetry both in the ventricles and in the heart as a whole, indicates that their development follows the most general laws of morphogenesis. Anthropometric studies of the anatomical structures of the fetal heart chambers were carried out in order to identify and describe the harmonious proportions between them. By analogy with the three-member kinematic blocks of the musculoskeletal system, three values of the measured anatomical structures were used to 
calculate the wurf between the parameters inside the heart chambers, taking into account the age of the fetus and the shape of the heart. Used according to the formula for calculating the wurf: a) the width and height of the right atrium, the length of the distance between the openings of the vena cava; b) the size of the area of the openings of the vena cava and the right atrioventricular opening; c) the length of the sinus and trabecular parts of the right ventricle (in the inflow and outflow section). The numerical values of these parameters can be represented as a wurf (wurf proportion). Regardless of the age and shape of the heart, the Wurf values are grouped around the value 1.3 (Table 5).

Table 5. Values of wurfs (W) of a three-membered block consisting of the width of the right atrium, its height and the length of the distance between the openings of the vena cava in human fetuses 13 - 36 weeks with different heart shapes, rel. units

\begin{tabular}{|l|l|l|l|}
\hline $\begin{array}{l}\text { Fetal age } \\
\text { (weeks) }\end{array}$ & \multicolumn{2}{|l|}{ Wurf value, rel. } & \multicolumn{2}{l|}{} \\
\hline & Narrow and long heart & Intermediate heart shape & Wide and short heart \\
\hline $13-16$ & 1,29 & 1,28 & 1,23 \\
\hline $17-20$ & 1,30 & 1,30 & 1,31 \\
\hline $21-24$ & 1,33 & 1,31 & 1,40 \\
\hline $25-28$ & 1,30 & 1,28 & 1,27 \\
\hline $29-32$ & 1,28 & 1,32 & 1,40 \\
\hline $33-36$ & 1,34 & 1,34 & 1,28 \\
\hline
\end{tabular}

The average value of the Wurf proportion of the area of the holes of the superior and inferior vena cava and the area of the right atrioventricular foramen in fetuses with a narrow long heart was 1.308; in hearts of a transitional form - 1.270; for wide short hearts 1.318. The wurf value of the length of the sinus part, trabecular part (in the inflow and outflow sections) of the right ventricle in the heart of the transitional form varied from 1.23 to 1.29. Thus, the area of the openings of the vena cava, the right atrioventricular opening, by analogy with the three-membered kinematic blocks of the musculoskeletal system, can be represented as a three-membered block. Similarly, the width and height of the right atrium and the distance between the openings of the vena cava, the length of the sinus and trabecular parts of the right ventricle (in the inflow and outflow sections) are related to each other. The calculations showed that the digital values of the wurfs of the area of the 
openings of the vena cava, the right atrioventricular opening in fetuses of 13 - 36 weeks, regardless of age and heart shape, are close to 1.309 ("golden wurf").

The relationship between the area of the openings of the vena cava and the right atrioventricular opening forms a harmonious wurf proportion, close to the value of 1.31. The wurf value between the parameters of the right atrium and the length of the distance between the openings of the vena cava is also close to 1.31. In fetuses of different ages, the growth of the heart occurs with the preservation of the size of the wurf of each indicated threemembered block. Anthropometric studies based on measurements of the proportions of three anatomical structures open up great opportunities. Subordination to the laws of conformal symmetry is manifested not only in the relationship between the parameters of the heart itself at all age periods, but also in the relationship of anatomical structures inside the heart chambers. The numerical values of the wurfs of the human heart can serve as an objective indicator of the harmony of its proportions. "Golden Wurf" is a three-member canon of not only the human body as a whole, his musculoskeletal system, but also the heart at all stages of individual development.

\section{Conclusion}

Based on the analysis of the quantitative anatomy of the heart and its chambers, it seems possible to conclude that two types of proportions are realized in the structure of the organ: affine (simple) relations of two anatomical measurements of the heart chambers and Wurf proportions. In the human heart, both within the same age and in different age periods, the proportions of ratios between the parameters of the ventricles are widely implemented in the range between 1.2 - 1.3. Subordination to the laws of conformal symmetry is manifested not only in the relationship of the parameters of the heart itself at all age periods, but also in the relationship of anatomical structures inside the chambers of the heart. In this work, the processes of heart formation are considered on the basis of the laws of conformal symmetries. Плоскость при этом интерпретируется как частный случай сферы бесконечно большого размера. The principle of conformal symmetry is reflected in the construction of the ventricles and the heart as a whole, in the structure of the kidneys, lungs, and permanent teeth of an adult (Spirina G.A., 2017, 2018). It is the most general principle of morphogenesis, determines the unity in the variety of forms of organs. Three segments of the measured anatomical parameters are used to calculate conformal symmetries. The presented data on the 
subordination of heart formations to the laws of conformal symmetry should be considered in close connection with their individual and age-related variability.

\section{Conclusions:}

1. In the structure of the heart, two types of proportions are realized: affine (simple) relations of two anatomical dimensions of the heart chambers and Wurf proportions.

2. Subordination to the laws of conformal symmetry is manifested not only in the relationship between the parameters of the heart itself at all age periods, but also in the relationship of anatomical structures inside the heart chambers.

3. The presented data on the subordination of heart formations to the laws of conformal symmetry should be considered in close connection with their individual and age-related variability.

\section{References}

[1] Nigmatullin R.T., Gafarov V.G., Salikhov A.Yu. The soft skeleton of the human face. Ufa. 2003.136 p.

[2] Petukhov S.V. Biomechanics, Bionics and Symmetry. Moscow: Nauka, 1981, 238 p.

[3] Petukhov S.V. Higher symmetries, transformations and invariants in biological objects // System, symmetry, harmony. - M .: Mys1, 1988. - S. 260 - 274.

[4] Petukhov S.V. Geometries of living nature and algorithms of self-organization // Mathematics and Cybernetics. Publishing house "Knowledge". M., 1988. - No. 6. - P. 3 29.

[5] Petukhov S.V. Symmetries in biology // Appendix to the book: A.V. Shubin, V.A. Koptsik "Symmetry in Science and Art", 3rd edition, M., 2004. - pp. 489 - 546.

[6] Shmalgauzen I.I. Definition of basic concepts and methods for studying growth. // Animal growth / ed. Kaplansky S.Ya., Mitskevich M.S., Tokin B.P., Shmalgauzen I.I. M., L .: Biomedgiz, 1935 .-- S. 8 - 60.Spirina G. A. Realization of the Principle of Conformal Symmetry in the Structure of the Heart // Advances in Human Factors in Simulation and Modeling. Proceeding of the AHFE International Conference of Human Factors in Simulation and Modeling/ Los Angeles, California, USA. Springer, 2017. Vol. 26. - P. $175-183$. 
[7] Spirina G. Comparative analysis of the quantitative parameters of the different shapes of the heart in human fetuses // Intelligent Human Systems Integration. Proceeding of the 1 - st International Conference on Intelligent Human Systems Integration (IHSI 2018): Integrating People and Intelligent Systems. Dubai. United Arab Emirates. Springer. 2018. -6 p. 